\title{
The Mobile Water Quality Monitoring System Based on Low- Power Wide Area Network and Unmanned Surface Vehicle
}

\author{
Wei Chen, ${ }^{1}$ Xiao Hao, ${ }^{2}$ Kui Yan $\mathbb{D}^{1},{ }^{1}$ JianRong Lu, ${ }^{3}$ Jin Liu, ${ }^{1}$ ChenYu He, ${ }^{2}$ Feng Zhou, ${ }^{1}$ \\ and $\mathrm{Xin} \mathrm{Xu}^{2}$ \\ ${ }^{1}$ Industrial Center/School of Innovation and Entrepreneurship, Nanjing Institute of Technology, Nanjing, Jiangsu 211100, China \\ ${ }^{2}$ Graduate School, Nanjing Institute of Technology, Nanjing, Jiangsu 211100, China \\ ${ }^{3}$ Jiangsu Aviation Vocational and Technical College, Zhenjiang, Jiangsu 212134, China
}

Correspondence should be addressed to Kui Yan; yankui@njit.edu.cn

Received 12 August 2021; Revised 26 September 2021; Accepted 27 September 2021; Published 21 October 2021

Academic Editor: Yuyu Yin

Copyright (c) 2021 Wei Chen et al. This is an open access article distributed under the Creative Commons Attribution License, which permits unrestricted use, distribution, and reproduction in any medium, provided the original work is properly cited.

\begin{abstract}
The increasingly serious water pollution problem makes efficient and information-based water quality monitoring equipment particularly important. To cover the shortcomings of existing water quality monitoring methods, in this paper, a mobile water quality monitoring system was designed based on LoRa communication and USV. In this system, the USV carrying water quality sensors was used as a platform. Firstly, the LoRa network is used to monitor water quality over a large area. Secondly, the unmanned surface vessel controls the position error within $\pm 20 \mathrm{~m}$ and the velocity error within $\pm 1 \mathrm{~m} / \mathrm{s}$ based on the Kalman filter algorithm. Thirdly, the genetic algorithm based on improved crossover operators is used to determine the optimal operational path, which effectively improves the iterative efficiency of the classical genetic algorithm and avoids falling into local convergence. In the actual water surface test, its packet loss probability within a working range of $1.5 \mathrm{~km}$ was below $10 \%$, and the USV could accurately navigate according to the preset optimal path. The test results proved that the system has a relatively large working range and high efficiency. This study is of high significance in water pollution prevention and ecological protection.
\end{abstract}

\section{Introduction}

Today, the water quality in coastal and inland lakes is deteriorating under the influence of increasing human social and economic activities. The ill-being water environment has caused irreparable losses to human health, production, and living, so that the protection of the water ecological environment demands immediate attention [1].

The data collection and monitoring of the water area take an important part in the protection and management decision-making of the water ecosystem. In spite of the research achievements, several problems in the field of water quality monitoring still need to be tackled.

(i) Monitoring scope: for large natural reserves of hundreds or even thousands of square kilometers, expensive economic costs will be incurred if monitoring and sensing device is arranged.

(ii) Remote location of the monitored area: special communication infrastructure should be erected for monitoring device in such areas.

(iii) Daily maintenance of device: a traditional monitoring device needs manual inspection and maintenance one by one, which will cause high human resource costs [2]. To address these issues, this paper investigates efficient water quality monitoring methods.

\section{Related Works}

There are three main approaches to current water quality monitoring: labor-intensive manual sampling, construction 
of fixed monitoring stations in monitoring waters, and mobile water quality monitoring through autonomous robots such as unmanned boats. Current water quality monitoring methods based on the second approach are many; for example, Ruan and Tang combined solar charging and wireless sensor network technology to design an energy-saving low-carbon water quality monitoring system [3]. Nam et al. designed a wireless sensor network system based on CDMA (Code Sector Multiple Access) and ZigBee technology when monitoring the water environment inhabited by coastal fish [4]. Wiebke et al. [5] deployed a wireless sensor network in coastal fishing grounds with low-cost compact buoys equipped with water quality monitoring sensors to analyze the impact of water quality parameters on aquaculture. These methods above have significant drawbacks; they can only monitor fixed locations and still require manual repositioning of node locations if the monitoring target is to be changed.

In response to above drawbacks, many studies have been made on the application of unmanned vessels for water quality monitoring. Cao et al. designed a 4G technology-based automatic navigation water quality monitoring unmanned boat, which can navigate to the preset monitoring point and collect water quality information at the location [6]. Siyang and Kerdcharoen realized the upload and storage of water quality monitoring data from unmanned boat based on Zigbee network, and the effective monitoring distance is about within $300 \mathrm{~m}$ [7]. Yang et al. in Taiwan designed a double-hulled water quality monitoring unmanned boat, which is stable and able to conduct water sampling on the basis of water quality collection. Bălănescu et al. combined blockchain technology with unmanned boat water quality monitoring work, providing a high security data collection, transmission, and management scheme [8]. However, these current research results are either limited by the shortcomings of ZigBee or WiFi, such as short communication distance and weak antiinterference ability, or limited by the high power consumption of $4 \mathrm{G}$ or GPRS, and the need for additional fees.

To cope with these existing problems, the emerging LoRa technology that boasts low cost, long communication distance, and strong endurance was introduced in this paper [9]. Further, the USV can navigate autonomously on the water surface, by which the efficiency of water surface working can be enhanced to a great degree [10]. In this paper, a mobile water quality monitoring system that works based on USV and LoRa was designed to effectively cover the shortcomings of existing methods. Featuring good energy-saving performance, low cost, and wide monitoring range, this system can be used to collect and monitor the data of the target water area by any environmental protection agency and individual, presenting extremely important research significance in the prevention and treatment of water pollution and the construction of a good aquatic ecology.

\section{Architecture of the Mobile Water Quality Monitoring System}

The mobile water quality monitoring system proposed in this paper integrates path planning, autonomous navigation, real-time water quality monitoring, and remote monitoring. This system is composed of parts: shipboard system, LoRa gateway, and monitoring terminal. The system architecture is shown in Figure 1.

First, the shipboard system mainly consists of positioning and navigation system, power system, LoRa communication system, and main control board. It has the functions of obtaining GPS positioning coordinates, reading heading, and speed information of unmanned surface vessels. The shipboard system interacts with the shore-based monitoring platform for data commands through LoRa network and with the water quality monitoring system for control commands through RS232 interface. The shipboard system according to the shore-based monitoring platform to send control commands for independent cruise or remote control action controls the water quality monitoring system for water quality testing.

Second, the water quality detection system consists of a main control board and four elements of water quality sensors such as temperature, turbidity, $\mathrm{pH}$, and conductivity. It receives the instructions that sent by the shipboard system to collect water body information on a regular basis.

Third, the shore-based platform is mainly developed based on windows operating system, including monitoring software programs and data processing algorithms. The host computer is mainly based on the human-computer interaction interface, completing LoRa communication, algorithm call, map display, data sending and receiving, and display functions; the algorithm application is mainly through the $\mathrm{C}++$ call MATLAB algorithm, sending the calculated operation path coordinates to the unmanned ship, so as to achieve the application of multipoint monitoring path planning.

The shipboard system of the USV is designed with a catamaran with a length of $0.8 \mathrm{~m}$, a width of $0.68 \mathrm{~m}$, and a maximum speed of $8 \mathrm{~km} / \mathrm{h}$. Structured with two parallel hulls of equal size, the catamaran has a wider beam and a shallower draft than a monohull, making the course of the entire ship more stable [11]. The structure of the unmanned surface vessel is shown in Figure 2.

Two DC motors are provided to serve as the kinetic drive device of the USV and coordinated with the motor driver; the hull steering can be adjusted through the differential control method. The USV is equipped with the positioning module ATK1218-BD and the attitude detection module MPU9250. The loose coupling integrated navigation algorithm was introduced to calculate the hull attitude and position. To meet the basic monitoring requirements, temperature, $\mathrm{pH}$, turbidity, and conductivity were selected as the monitoring elements in this paper. The E-201C pHwater temperature composite sensor, the TSW-30 turbidity sensor, and the DJS-12 conductivity electrode sensor are employed and paralleled via RS485 communication protocol, and these sensors can be replaced and configured according to actual use condition. STM32F103ZET6 acts as the central controller to complete data collection and calculation.

The LoRa communication technology used for USV can effectively expand the monitoring scope and save costs. In this paper, close attention was paid to the design of the ship-shore LoRa communication system and the path 


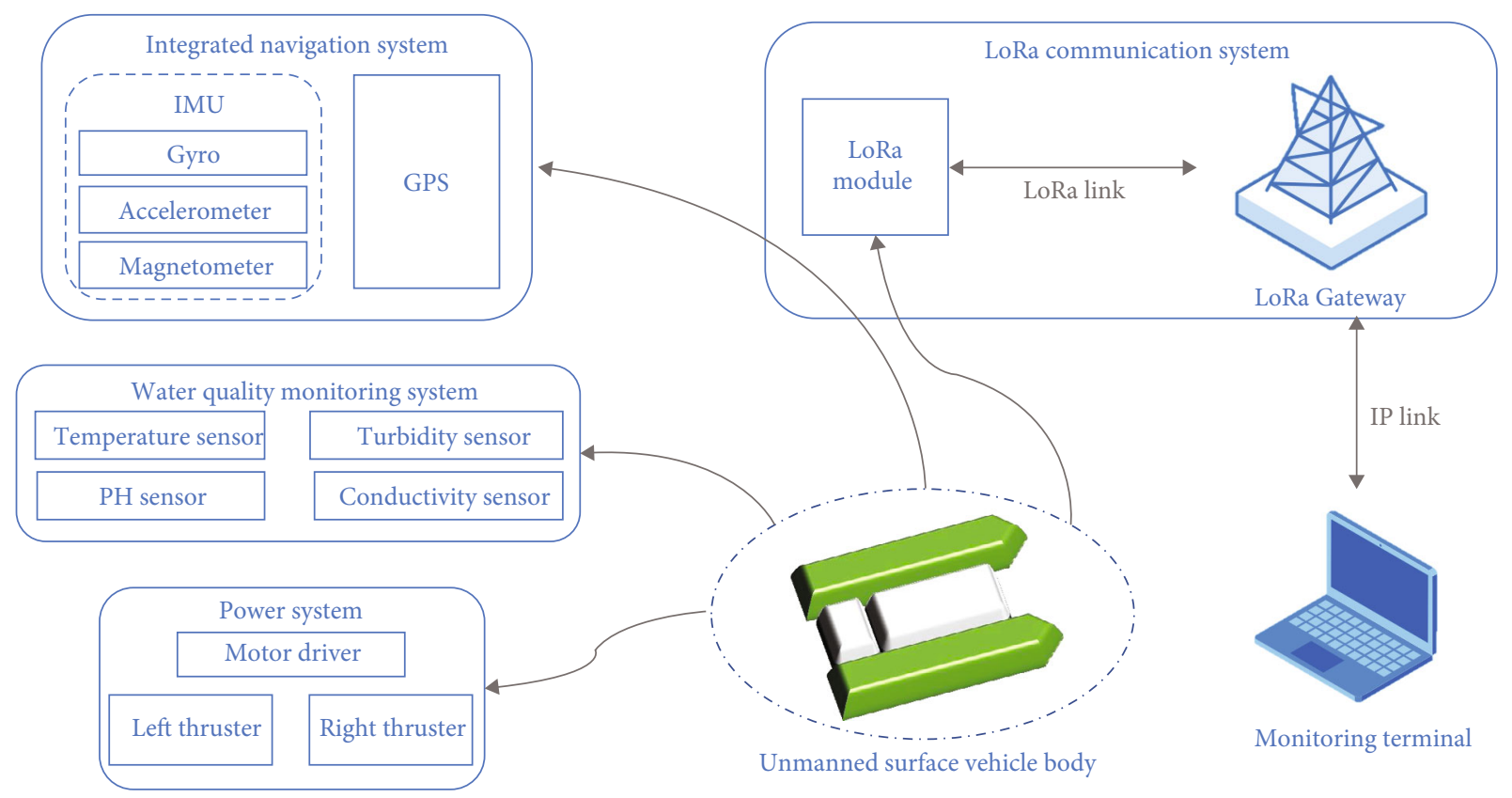

FIgURE 1: Architecture of the mobile water quality monitoring system.

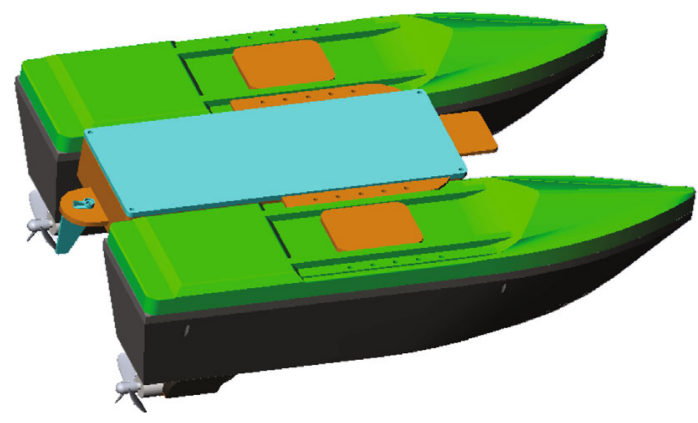

FIGURE 2: The mechanical structure of USV.

planning algorithm of USV was improved. In addition, ONENET_China Mobile IoT Development Platform-was used as the monitoring terminal of this system to help realize the device monitoring and configuration, real-time data monitoring, data storage, and other functions [12].

\section{LoRa Communication System}

\subsection{Shipboard LoRa Nodes}

4.1.1. Communication Node Hardware. This system is designed with LoRa radio frequency module to receive and transmit the water quality data. The programming was conducted in the MCU to control the LoRa module and send the encoded information through the module. The integrated $+20 \mathrm{dBm}$ power amplifier is provided to ensure that the long-distance wireless communication is available under the condition of sensitivity as low as $-148 \mathrm{dBm}$. The circuit schematic diagram of the LoRa communication module is shown in Figure 3.
4.1.2. Communication Node Software. In this study, the control program of the STM32 single-chip microcomputer was designed in the Keil integrated development environment and finally programmed to the MCU to complete the development after compilation, simulation, and debugging. The program was written to complete such functions as data collection, signal conversion, and uploading data. The flow of node program is shown in Figure 4. After the program starts, it is divided into the following steps: first, initialize the device and make the LoRa module connect to the network; second, enter the main program and wait for the timer to trigger the detection task; third, complete the trigger and collect the water body data; fourth, carry out MCU data processing and ADC conversion; fifth, store the data in the buffer after processing is completed and wait for sending; sixth, after sending, enter the sleep state and wait for the timer to trigger the detection command again.

\subsection{Shore-Based LoRa Gateway}

4.2.1. Gateway Hardware. The embedded gateway designed in this study was composed of an industrial-control core board, a LoRa gateway module, and a 4G LTE mobile data board, as shown in Figure 5.

In order to cope with large-scale distributed monitoring scenarios, it is necessary to extend the downlink channel of gateway. A symmetrical channel processor refers to the coexistence of multiple SX1278s by combiners based on a communication channel provided by the existing SX1278 RF chip. Connect the module with the serial port of LoRa gateway, and control frequency band, power, spreading factor, and other RF parameters of each channel by application program to operate the serial port, and fix all channels of modules as downlink channels. This makes up for the lack 


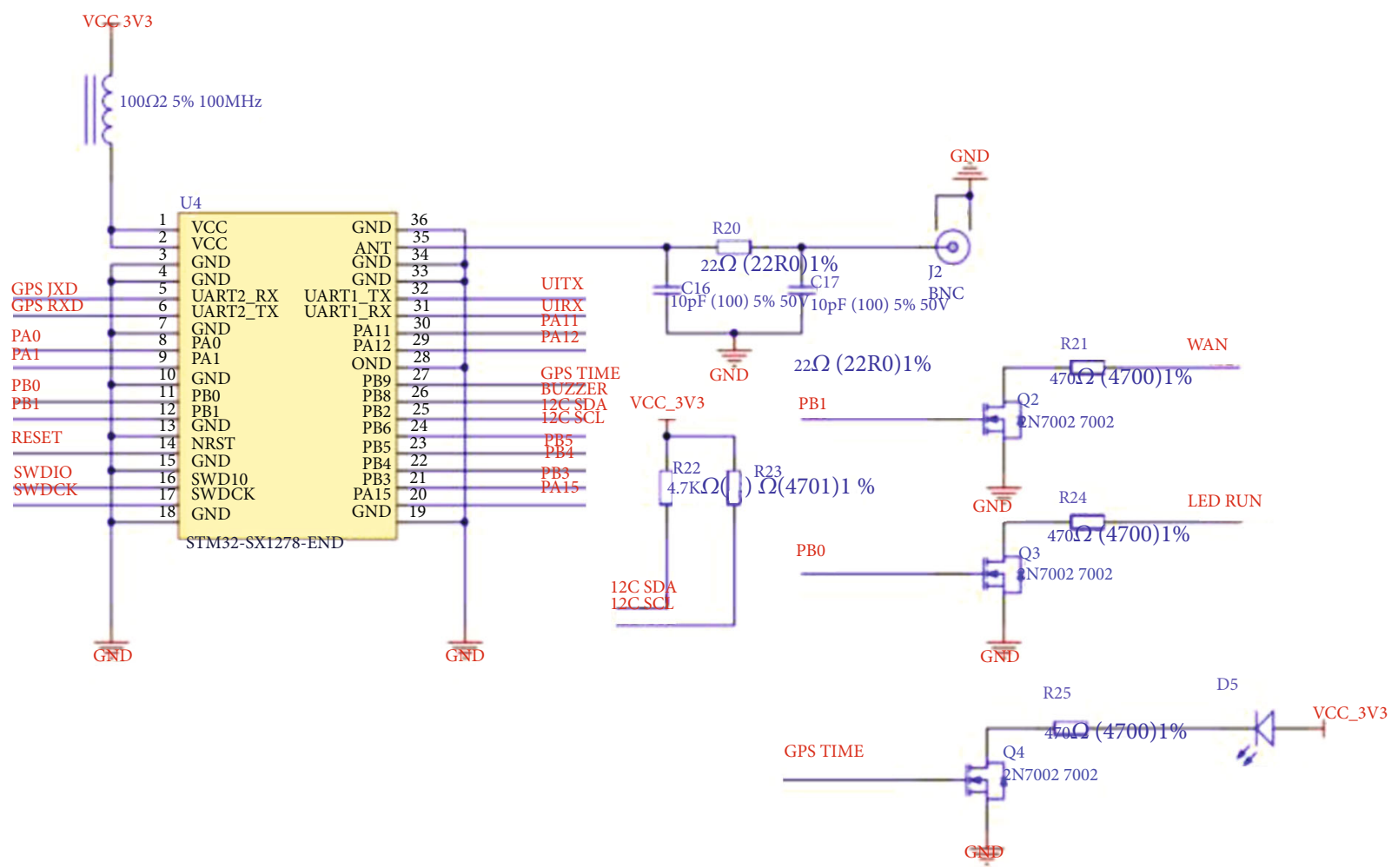

Figure 3: Schematic diagram of LoRa RF module.

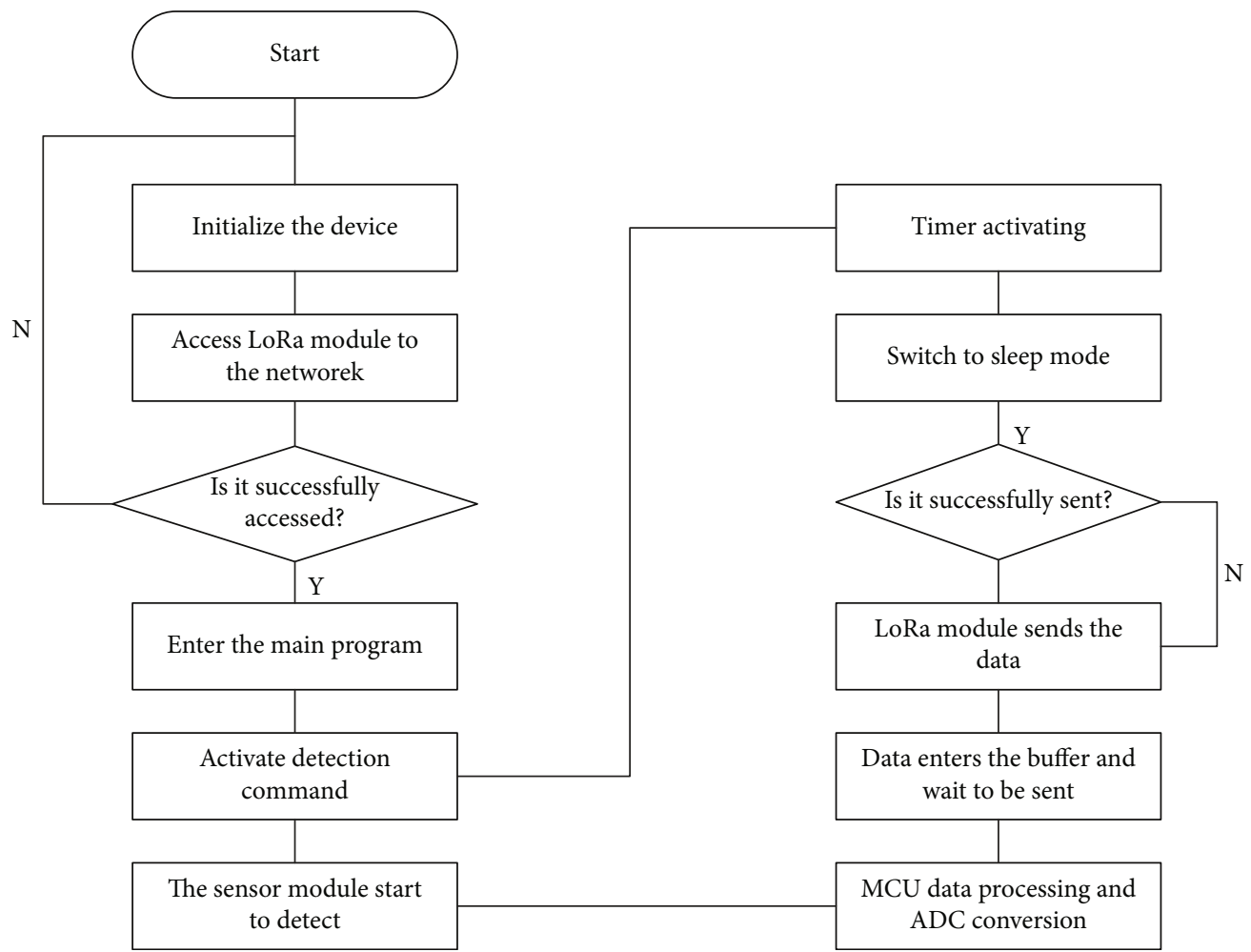

Figure 4: Flow of sensing layer node program. 


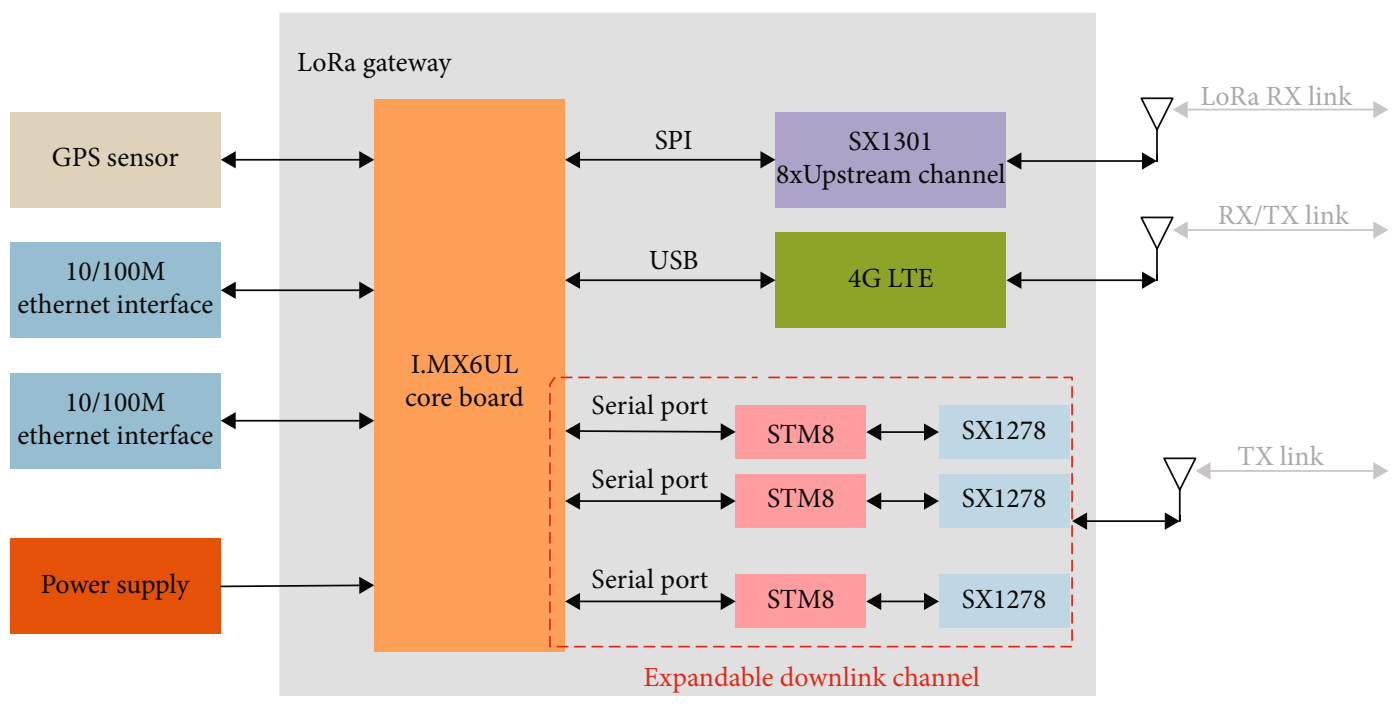

FIgURE 5: Architecture of gateway hardware.

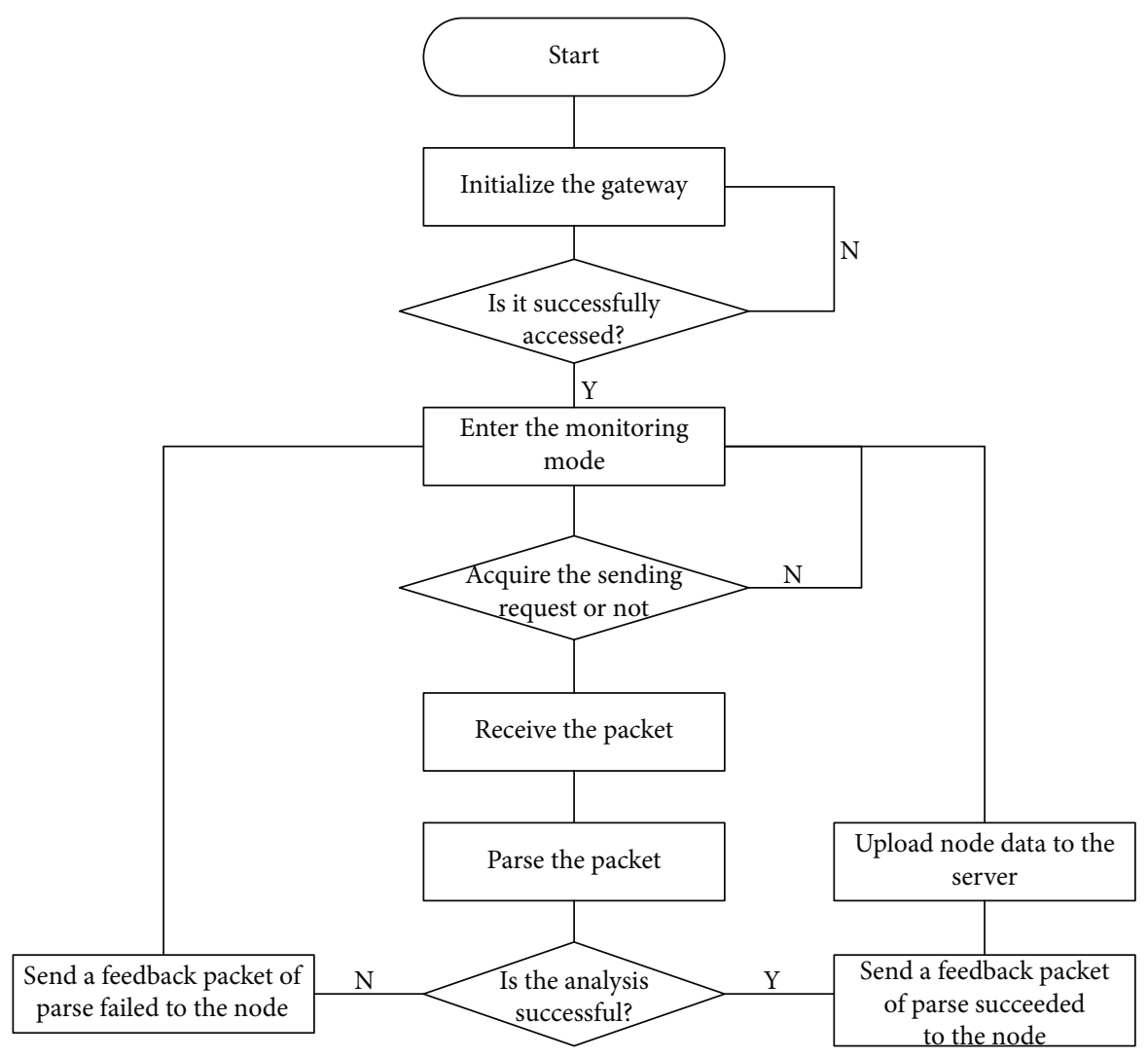

FIGURE 6: Flow of gateway program.

of one downlink channel of existing LoRa gateway and realizes a LoRa gateway structure under multichannel symmetrical channel, which can alleviate transmission congestion and packet loss caused by large-scale data access.

4.2.2. Gateway Software. The workflow of the gateway in this study is shown in Figure 6. After the gateway program starts, it is divided into the following steps: first, initialize the gateway device and check whether the initialization is successful; second, the gateway enters listening mode; third, determine whether to obtain the send request; fourth, receive the packet after successfully obtaining the send request, otherwise continue listening; fifth, parse the packet and determine whether the parsing is successful; sixth, if the parsing is 


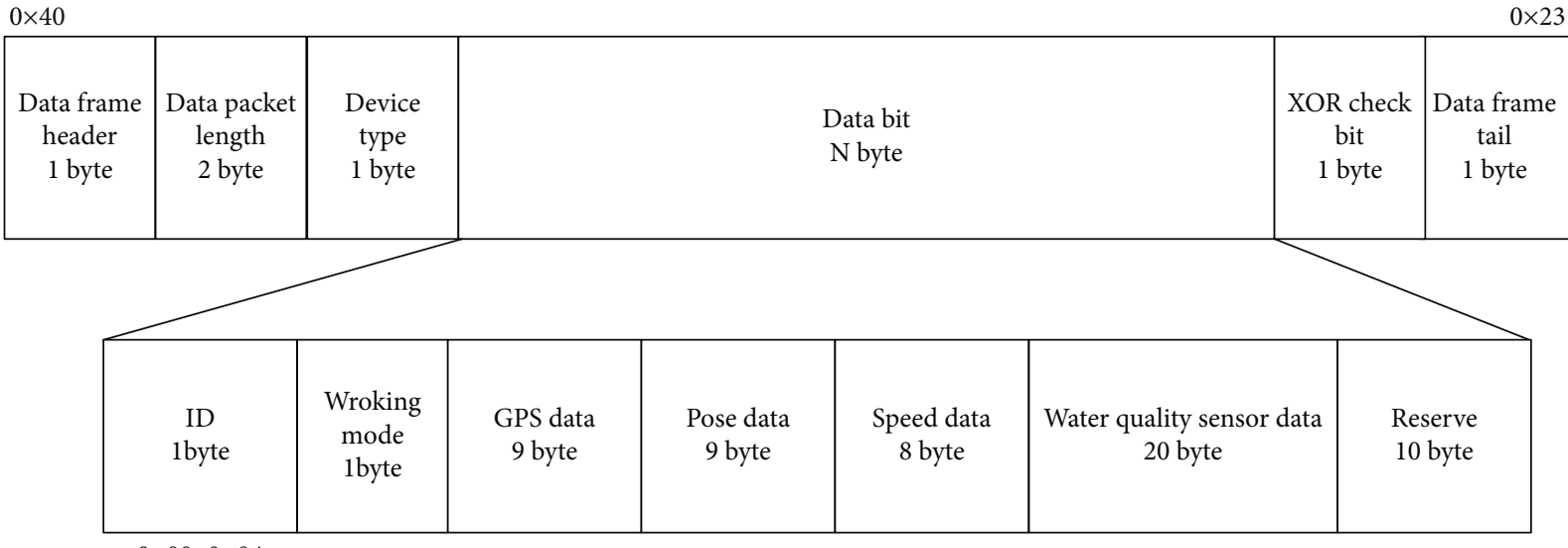

Figure 7: Format of protocol frame sent by monitoring terminal.

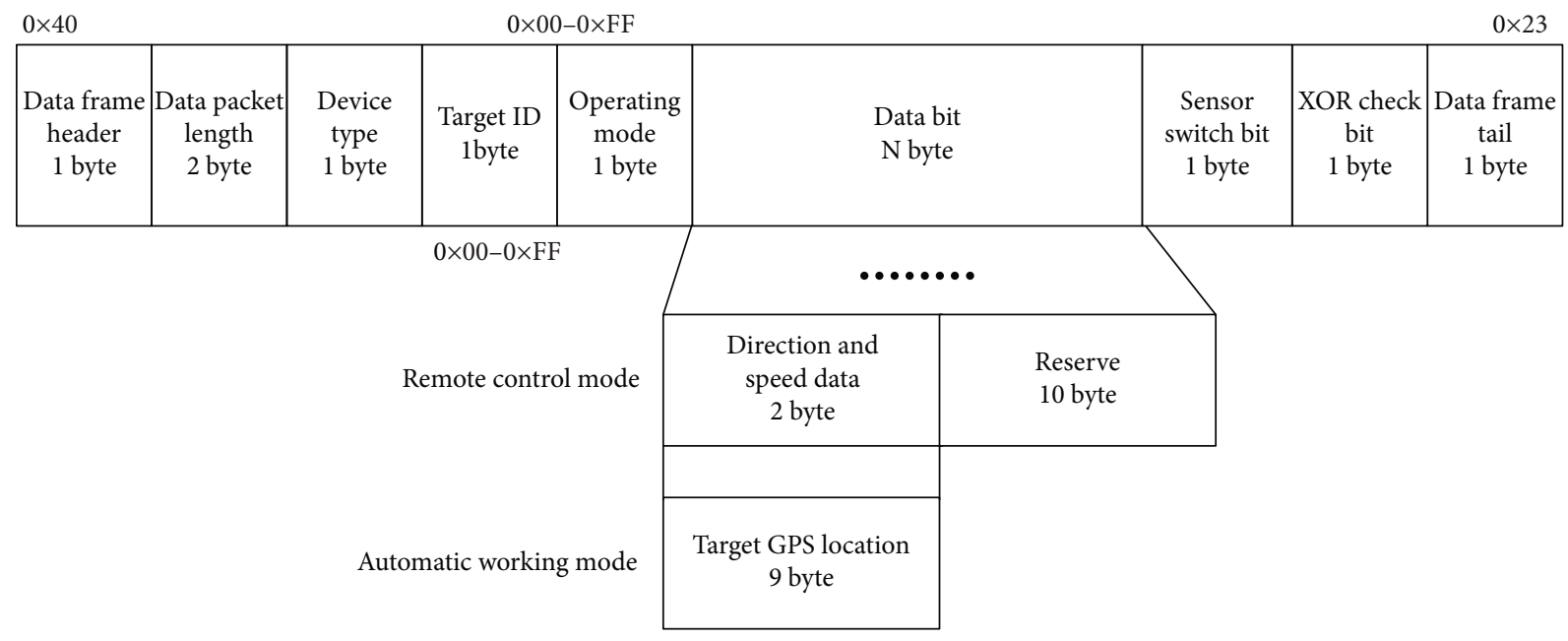

FIgURE 8: Flow of gateway program.

successful, upload the data to the user monitoring platform Otherwise, send the feedback packet to the node for failed parsing.

\subsection{Ship-Shore Interaction LoRa Communication Protocol.} The parsing of the communication protocol by the onboard system is mainly implemented by a finite state machine. We preset three states: stop, autonomous, and remote control. When the shipboard system receives the protocol frame from the shore-based monitoring platform, it parses the operation mode field in the protocol. The finite state machine further processes the data in the data frame by the different operational states. In the case of remote control mode, the speed and heading information in the data frame is parsed and the remote control procedure is executed. In the case of autonomous mode, the GPS coordinates of the target point in the data frame are resolved. Then, the heading is calculated based on the current GPS coordinates, and navigation to the target point is performed. In case of stop mode, the operation is terminated. During the operation of the unmanned surface vessel, water body data and navigation status are regularly collected and uploaded to the user monitoring platform. The user monitoring platform receives the data packets and parses, displays, and stores them.

4.3.1. USV Node Sends Protocol Frame. The communication when the USV node sends data to the LoRa gateway was in line with the protocol frame format shown in Figure 7.

This protocol frame contains 64 bytes, composed of data frame header, data packet length, device type, target ID number, operating mode, GPS data, pose data, speed data, water quality sensor data, reserved bits, XOR check bit, and data frame tail.

4.3.2. Protocol Frame Sent by Monitoring Terminal. The communication when the monitoring terminal sends data to the USV node was in line with the protocol frame format shown in Figure 8.

The protocol frame contains 21-28 bytes, composed of data frame header, data packet length, device type, target ID number, operating mode, direction and speed data, target GPS position, reserved bit, sensor switch bit, XOR check bit, and data frame tail. 


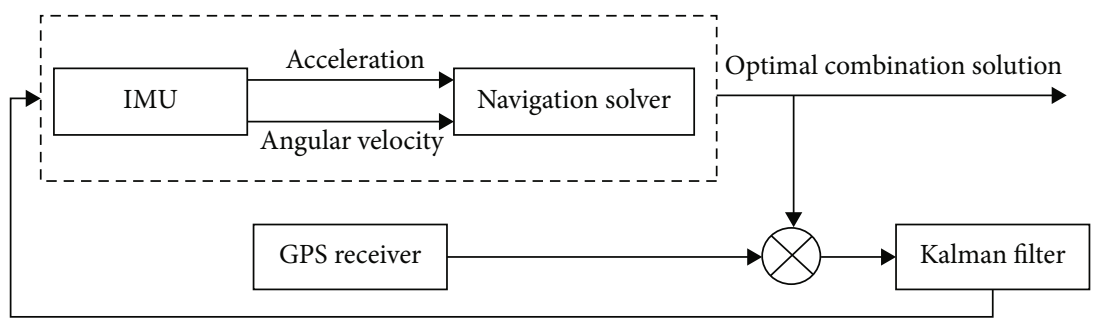

FIGURE 9: Improved combination method.

The protocol frame is a data frame with variable length. When the remote control mode is selected as the working mode, the direction and speed data were transmitted in the data bit, and when the automatic working mode was selected, the target GPS location data were transmitted in the data bit.

\section{Integrated Navigation Method for USV}

For water quality monitoring cruise operations, the navigation algorithm is crucial. GPS navigation is vulnerable to interference from the external environment and limited by the low update frequency. It cannot meet the demand for continuous and stable positioning. The inertial navigation method calculates position and velocity by direct integration of inertial module measurement information data, and the accumulated error will occur and gradually increase with time. To address the above problems, we use an improved integrated navigation method based on the Kalman filter to achieve the fusion of GPS and inertial data. It can overcome the shortcomings of inertial sensor dispersion over time. The inertial sensors provide the acceleration and angular velocity of the ship, and the speed and position information of the ship can be calculated using the laws of physics. The Kalman filtering algorithm reduces the effect of cumulative errors while reducing the complexity of implementation. This improved integrated navigation method has the advantages of simple structure and small computational effort, which is well suited for low-cost unmanned ship navigation applications. Its schematic block diagram is shown in Figure 9.

Neglecting the velocity error and position error of the sky direction, the state variables of the combined navigation are:

$$
X=\left[\varphi_{E}, \varphi_{N}, \varphi_{U}, \partial V_{E}, \partial V_{N}, \partial L, \partial \lambda, \varepsilon_{b x}, \varepsilon_{b y}, \varepsilon_{b z}, \Delta_{x}, \Delta_{y}, \Delta_{z}\right]^{T} \text {, }
$$

where $\varphi_{E}, \varphi_{N}, \varphi_{U}$ denote the platform angle error in the east, north, and sky directions, respectively; $\partial V_{E}, \partial V_{N}$ denote the velocity error in the east and north directions, respectively; $\partial L, \partial \lambda$ denote the longitude and latitude error, respectively; $\varepsilon_{b x}, \varepsilon_{b y}, \varepsilon_{b z}$ denote the constant drift of gyroscope in the east, north, and sky directions, respectively; and $\Delta_{x}, \Delta_{y}, \Delta_{z}$ denote the constant drift of acceleration in the east, north, and sky directions, respectively.

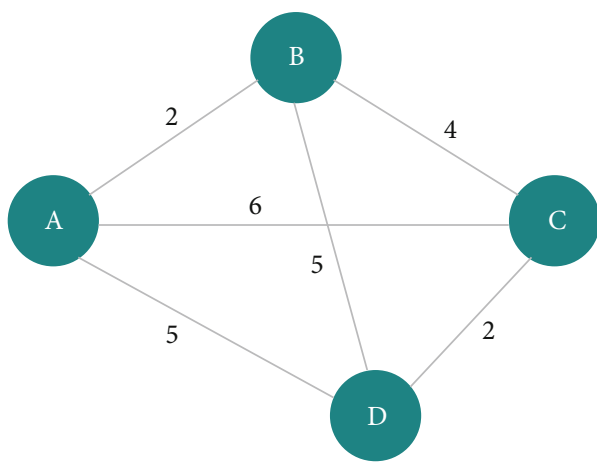

FIgURE 10: Example of weighted completely undirected graph for TSP problem.

$1|3| 5|6| 7|4| 2$

FIGURE 11: Chromosome representation method.

The system noise vectors are

$$
W=\left[\omega_{\varepsilon E}, \omega_{\varepsilon N}, \omega_{\varepsilon U}, \omega_{a E}, \omega_{\mathrm{aN}}, 0,0,0,0,0,0,0,0\right]^{T},
$$

where $\omega_{\varepsilon E}, \omega_{\varepsilon N}, \omega_{\varepsilon U}$ denote the random drift of the gyroscope in the east, north, and sky directions, respectively, and $\omega_{a E}, \omega_{\mathrm{aN}}$ denote the random drift of the accelerometer in the east and north directions, respectively.

Assume that the gyroscope and accelerometer drift obey Gaussian distribution, that is,

$$
\begin{gathered}
\varepsilon=0, \Delta=0, \\
E\left[W(t) W(t)^{T}\right]=Q(t) .
\end{gathered}
$$

Also considering the unmanned ship work actual, the system works in the horizontal plane; then, the system state equation is

$$
\begin{aligned}
\phi_{\bar{E}}= & -\frac{\partial V_{N}}{R_{m}+h}+\left[\omega_{i e} \sin L+\frac{V_{E}}{R_{n}+h} \tan L\right] \phi_{N} \\
& -\left[\omega_{i e} \cos L+\frac{V_{E}}{R_{n}+h} \tan L\right] \phi_{U}+C_{b}^{t}(1,1) \varepsilon_{b x} \\
& +C_{b}^{t}(1,2) \varepsilon_{b y}+C_{b}^{t}(1,3) \varepsilon_{b z}+\omega_{\varepsilon E},
\end{aligned}
$$




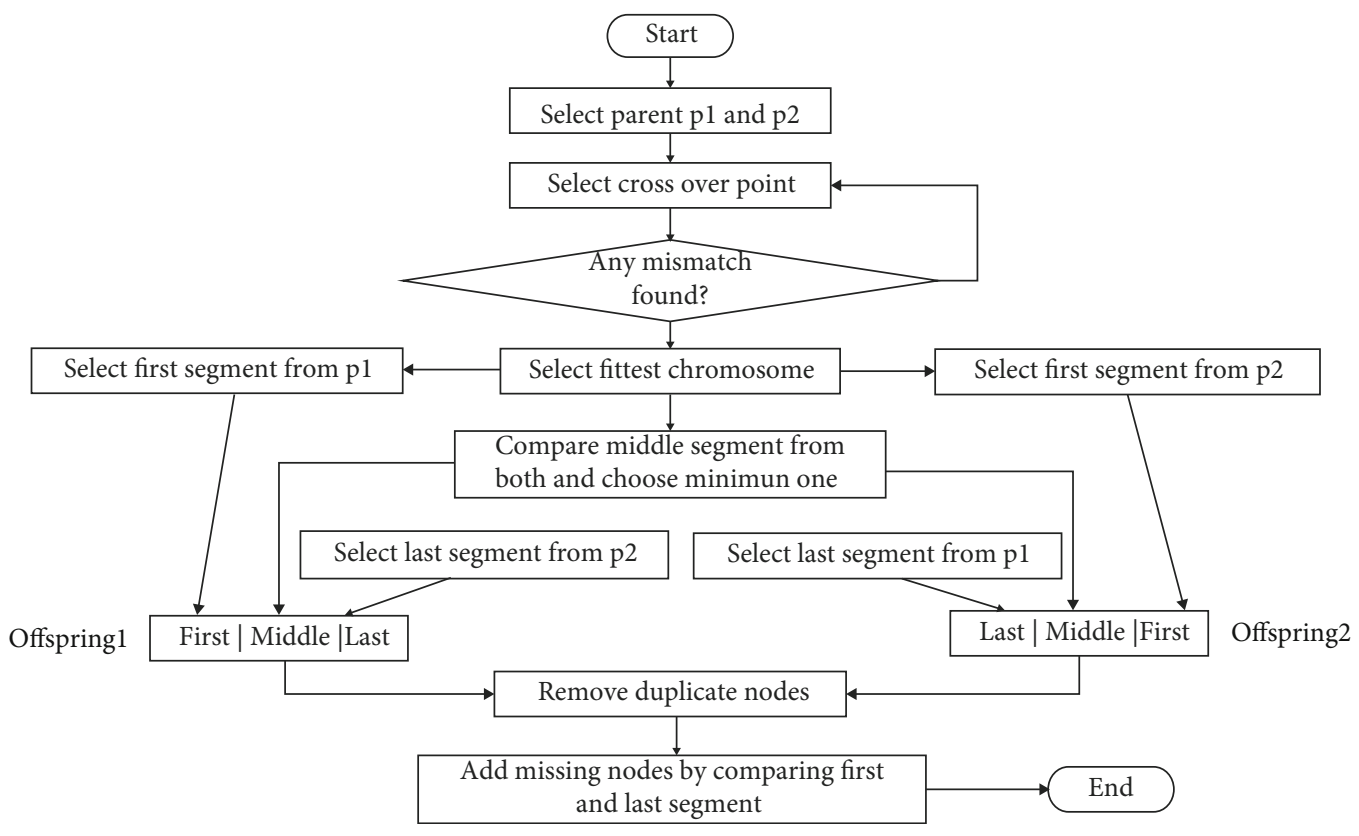

FIgURE 12: Cross process diagram.

$$
\begin{aligned}
\phi_{N}= & \frac{\partial V_{E}}{R_{n}+h}-\omega_{i e} \sin L \partial L-\left[\omega_{i e} \sin L+\frac{V_{E}}{R_{n}+h} \tan L\right] \phi_{E} \\
& -\frac{V_{N}}{R_{m}+h} \phi_{U+C_{b}^{t}(2,1) \varepsilon_{b x}+C_{b}^{t}(2,2) \varepsilon_{b y}+C_{b}^{t}(2,3) \varepsilon_{b z}+\omega_{e N}} \\
\phi_{U}= & {\left[\omega_{i e} \cos L+\frac{V_{E}}{R_{n}+h}\right] \phi_{E}+\frac{V_{E}}{R_{m}+h} \phi_{N}+\frac{\tan L}{R_{n}+h} \partial V_{E} } \\
& +\omega_{i e} \sin L \partial L+\left[\omega_{i e} \cos L+\frac{V_{E}}{R_{n}+h} \sec ^{2} L\right] \partial L \\
& +C_{b}^{t}(3,1) \varepsilon_{b x}+C_{b}^{t}(3,2) \varepsilon_{\mathrm{b} y}+C_{b}^{t}(3,3) \varepsilon_{b z}+\omega_{e U}
\end{aligned}
$$

$$
\begin{aligned}
\partial V_{E}= & {\left[2 \omega_{i e} \sin L+\frac{V_{E}}{R_{n}+h} \tan L\right] \partial V_{N}+\frac{V_{N}}{R_{m}+h} \tan L \partial V_{E} } \\
& +f_{U} \phi_{N}-f_{N} \phi_{U}-\left[2 \omega_{i e} \cos L V_{N}+\frac{V_{E} V_{N}}{R_{n}+h} \sec ^{2} L\right] \partial L \\
& +C_{b}^{t}(1,1) \Delta_{x}+C_{b}^{i}(1,2) \Delta_{y}+C_{b}^{t}(1,3) \Delta_{z}+\omega_{a E}
\end{aligned}
$$

$$
\begin{aligned}
\partial V_{N}= & 2\left[\omega_{i} \sin L+\frac{V_{E}}{R_{n}+h} \tan L\right] \partial V_{E}+f_{U} \phi_{E}-f_{E} \phi_{U} \\
& -\left[2 \omega_{i e} \cos L+\frac{V_{E}}{R_{n}+h} \sec ^{2} L\right] \partial L V_{E}+C_{b}^{t}(2,1) \Delta_{x} \\
& +C_{b}^{t}(2,2) \Delta_{y}+C_{b}^{t}(2,3) \Delta_{z}+\omega_{a E}
\end{aligned}
$$

$$
\partial L=\frac{1}{R_{m}+h} \partial V_{N}
$$

$$
\partial \lambda=\frac{\sec L}{R_{n}+h} \partial V_{E}+\frac{V_{E} \sec L \tan L}{R_{n}+h} \partial L,
$$

where $V_{E}, V_{N}$ indicate the speed of the unmanned ship in the east and north directions, respectively; $\omega_{i e}$ indicates the angular velocity of the earth's rotation; and $f_{E}, f_{N}, f_{U}$ indicate the specific force felt by the accelerometer in the east, north, and sky directions, respectively.

Combining the above equations, the system equation of state can be written as

$$
\widehat{X}(t)=F(t) X(t)+W(t)
$$

where $\widehat{X}(t)$ indicates the estimated state, $F(t)$ indicates the state matrix, $X(t)$ indicates the current state, and $W(t)$ indicates the noise vectors.

In the navigation system, there are two groups of observation equations: one group is the position observation value, which is the difference between the latitude and longitude information given by the inertial guidance system and the corresponding position information given by the GPS receiver; the other group of observation value is the difference between the velocity in each direction given by the two systems.

The position measurement information of INS can be expressed as the sum of the true value and the error:

$$
\left[\begin{array}{c}
L_{I} \\
\lambda_{I}
\end{array}\right]=\left[\begin{array}{c}
L_{t}+\partial L \\
\lambda_{\mathrm{t}}+\partial \lambda
\end{array}\right]
$$


TABLE 1: Example cost matrix.

\begin{tabular}{lccccccc}
\hline Node & 1 & 2 & 3 & 4 & 5 & 6 & 7 \\
\hline 1 & Inf & 29 & 82 & 46 & 68 & 52 & 15 \\
2 & 29 & Inf & 55 & 46 & 42 & 43 & 43 \\
3 & 82 & 55 & Inf & 68 & 63 & 20 & 23 \\
4 & 46 & 46 & 68 & Inf & 82 & 15 & 72 \\
5 & 68 & 42 & 63 & 82 & Inf & 74 & 23 \\
6 & 52 & 43 & 20 & 15 & 74 & Inf & 61 \\
7 & 15 & 43 & 23 & 72 & 23 & 61 & Inf \\
\hline
\end{tabular}

$$
\begin{aligned}
& \text { Offspring1 } 4|3| 5|7| 6|1| 2 \quad \text { Cost }=296 \\
& \text { Offspring2 } 4|5| 6|1| 3|7| 2 \quad \text { Cost }=356
\end{aligned}
$$

FIGURE 13: Representation of father generation chromosome.

$$
\begin{aligned}
& \text { Offspring1 } 4 \quad 4|3| 5|7| 6|7| 2 \\
& \text { Offspring2 } 4|5| 5|7| 6|1| 2
\end{aligned}
$$

FIGURE 14: Representation of the offspring chromosome obtained after Step 2.

Similarly, the position information of GPS can be expressed as the sum of the true value and the error:

$$
\left[\begin{array}{c}
L_{G} \\
\lambda_{G}
\end{array}\right]=\left[\begin{array}{c}
L_{t}+N_{N} \\
\lambda_{t}+N_{E}
\end{array}\right] .
$$

Then, the position observation equation is

$$
\begin{aligned}
& Z_{P}(t)=H_{P}(t) \mathrm{X}(t)+V_{P}(t), \\
& H_{P}(t)=\left[\begin{array}{ll}
0_{2 \times 5} & \operatorname{diag}\left[\begin{array}{ll}
1 & 1
\end{array}\right] 0_{2 \times 6}
\end{array}\right], \\
& V_{P}(t)=\left[\begin{array}{ll}
N_{N} & N_{E}
\end{array}\right] .
\end{aligned}
$$

Similarly, the velocity observation equation of INS is

$$
\begin{aligned}
\& Z_{V}(t) & =H_{V}(t) X(t)+V_{V}(t), \\
H_{V}(t) & =\left[\begin{array}{lll}
0_{2 \times 3} & \operatorname{diag}\left[\begin{array}{ll}
1 & 1
\end{array}\right] & 0_{2 \times 8}
\end{array}\right], \\
V_{V}(t) & =\left[\begin{array}{ll}
M_{N} & M_{E}
\end{array}\right] .
\end{aligned}
$$

By combining the position observation equation and the velocity observation equation, the observation equation of the combined INS/GPS navigation system can be obtained as

$$
Z(t)=\left[\begin{array}{l}
H_{P}(t) \\
H_{V}(t)
\end{array}\right] X(t)+\left[\begin{array}{c}
V_{P}(t) \\
V_{V}(t)
\end{array}\right]
$$

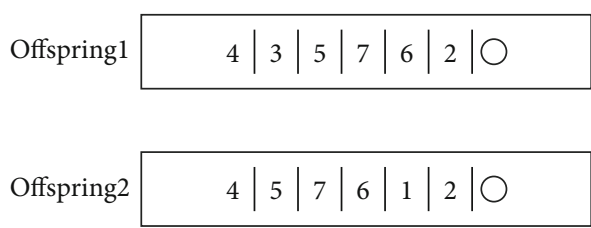

FIGURE 15: Representation of the offspring chromosomes after Step 3. O represents to the missing node to be supplemented.

$$
\begin{aligned}
& \text { Offspring1 } \quad \text { Cost = 287 } \\
& \begin{array}{r|c|c|c|c|c|c}
4 & 3 & 5 & 7 & 6 & 2 & 1 \\
\text { Offspring2 } & 4|5| 7|6| 1|2| 3 & \\
\end{array}
\end{aligned}
$$

FIGURE 16: Representation of the offspring chromosomes after Step 3.

\section{Study on USV Path Planning Based on Improved Genetic Algorithm}

6.1. Problem Description. Path planning is the key technology for a mobile water quality monitoring system to work efficiently. The available optional paths from the starting point to the end point in the water quality monitoring were countless. This paper is aimed at finding out the optimal working path with high efficiency and energy saving as indicators.

Based on the working characteristics of the USV traversing multiple monitoring points over the course of water quality monitoring, the water quality monitoring process was abstracted as the Traveling Salesman Problem (TSP) in this paper. Observe Figure 10; the essence of the problem is to find a Hamilton loop with the smallest weight in a weighted completely undirected graph.

The classic TSP could be described as follows: when the number of target cities $N$ and the distance between any cities are all known, the shortest path traversed all cities once and only once and returned to the starting city [13]. The problem can be described via the following mathematical model.

$$
\begin{gathered}
D=\left(d_{i j}\right)_{N \times N}\left\{\begin{array}{l}
d_{i j}, i \neq j, \\
\text { Inf }, i=j,
\end{array}\right. \\
\left\{\begin{array}{l}
\text { Tour }=\left\{T_{1}, T_{2}, \cdots, T_{p}, \cdots, T_{(n-1) !}\right\}, \\
n !=n \cdot(n-1) \cdots, \cdot 2 \cdot 1,
\end{array}\right. \\
\operatorname{Len}\left(T_{p}\right)=\left(\sum_{l=1}^{N-1} d_{T_{p(l)} T_{p(l+1)}}\right)+d_{T_{p(N)}{ }^{T} p(1)},
\end{gathered}
$$

where $N$ is the number of cities, $D$ is the distance matrix, $d_{i j}$ is the distance between two cities, Inf is a large enough positive number, $i, j$ are the city numbers, Tour is the set of paths, $T_{p(l)}$ is the $l$ th city in the path, and $\operatorname{Len}\left(T_{p}\right)$ is the total length of the path $T_{p}$. It can be seen that the optimal solution of TSP is the minimum value of solving $\operatorname{Len}\left(T_{p}\right)$. Then, an 


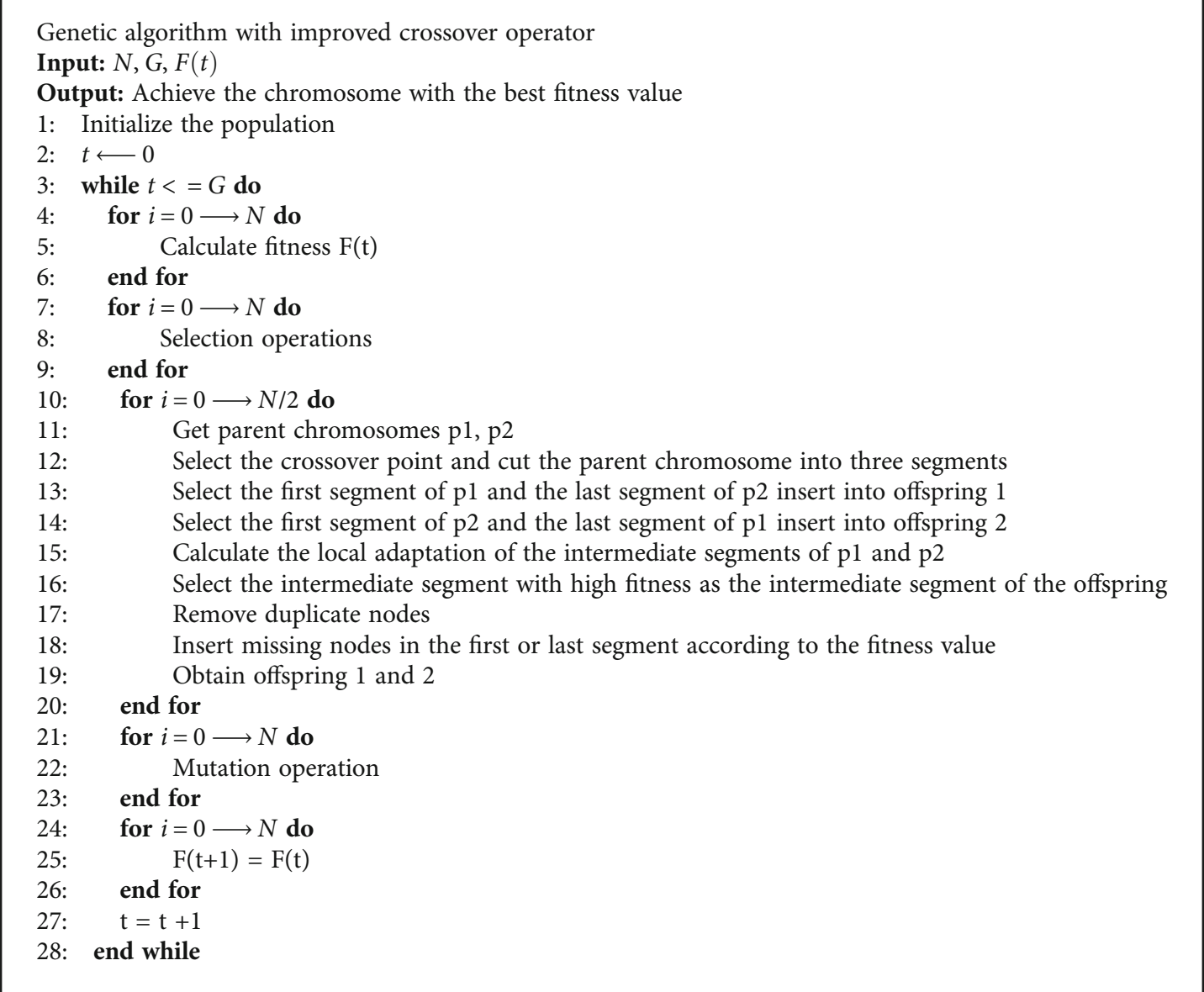

Algorithm 1: Pseudocode for the method proposed.

improved genetic algorithm was introduced in this paper to solve this problem.

6.2. Basic Genetic Algorithm Principle. In this paper, the basic genetic algorithm is improved so as to calculate the optimal path of the unmanned ship. The basic genetic algorithm is one kind of iterative algorithm, which is an intelligent computational model to complete the simulation of biological evolution process in nature with the help of computer technology. Compared with the traditional algorithms, the genetic algorithm not only has the characteristics of selflearning, self-organization, and self-adaptation but also has high robustness and wide applicability, which can effectively deal with the complex problems that are difficult to be solved by traditional optimization algorithms without the limitation of problem nature.

The main operators that manipulate chromosomes in genetic algorithms are as follows: population initialization operator, selection operator, crossover operator, and mutation operator. The population initialization operator prepares for the improvement of the feasible solution and the iterative process of the algorithm, mainly for the task of transforming the feasible solution of the problem into the corresponding chromosomes based on the coding scheme. The purpose of the selection operator is to select individuals of good breed. The operator is based on the evaluation of the fitness of the individuals and, according to a specific method, selects a part of the contemporary population with high fitness in preparation for the generation of the new generation. The crossover operator is an important operator that enables genetic algorithms to search for new solutions over a very wide space, in order to obtain new and better individuals. The crossover process is a process performed by a certain probability to replace and reorganize some genes of the chromosomes of the individuals already selected from the population into new individuals. The mutation operator ensures the diversity of individuals in the population, thus suppressing to some extent the problem of early convergence in the genetic algorithm.

The basic execution steps of the genetic algorithm are as follows:

(Step 1) In population initialization, first, complete the setting of the control parameters of the genetic algorithm, and then establish the first generation population using the population initialization operator.

(Step 2) Calculate the individual fitness. Construct the corresponding fitness function, and calculate the fitness of all individuals in the population according to the problem being addressed. 


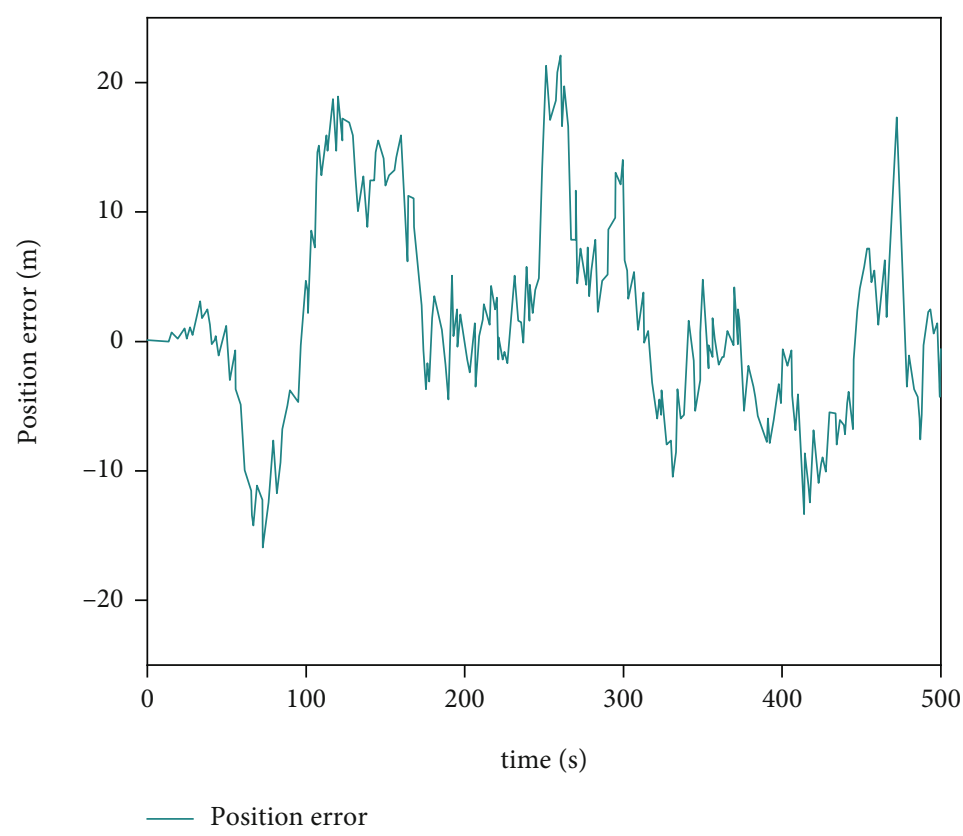

FIGURE 17: Position error convergence range after combined navigation filtering.

(Step 3) Determine whether to terminate the iteration. When the number of evolutionary generations is less than the maximum number of iterations, Step 4 is executed; otherwise, the iteration is terminated and the optimal individuals in the population are output.

(Step 4) Execute the selection operation. Use the selection operator to select some individuals from the population.

(Step 5) Execute the crossover operation. Compare the crossover probability, and perform the update operation on the selected individuals using the crossover operator.

(Step 6) Perform the variation operation. In contrast to the mutation probability, a new generation of population is obtained by performing the mutation operation on the selected individuals, updating the evolutionary generation and moving to Step 2.

In order to be able to make the offspring obtained by crossover jump out of the local optimal solution while retaining the excellent genes of the parent, this paper proposes an improved triple crossover operator, which divides the parent chromosome into three segments and then compares the recombination, so as to obtain better individuals.

6.3. Improved Genetic Algorithm. The high efficiency of the basic genetic algorithm is mainly attributed to its operators: replication, crossover, and mutation [14]. But the basic genetic algorithm is still exposed to such shorting such as poor local search ability and slow convergence speed [15]. In this paper, the iterative efficiency of genetic algorithm in

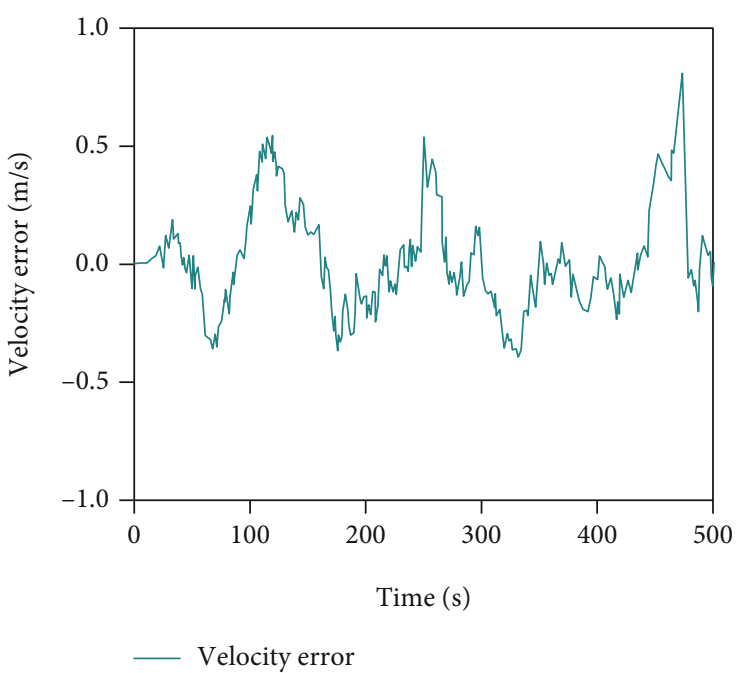

FIGURE 18: Velocity error convergence range after combined navigation filtering.

the path planning of USVs was lifted by improving the multipoint crossover operator.

In the process of species initializing, each chromosome was described as a series of nodes (each node was a monitoring target point). If there were seven monitoring target points in the job, the length of the chromosome would be set to 7 , as shown in Figure 11. The cost matrix could be used to calculate the distance cost between two monitoring target points.

The fitness function that used the basic genetic algorithm was reserved:

$$
F(x)=\frac{1}{f(x)}
$$




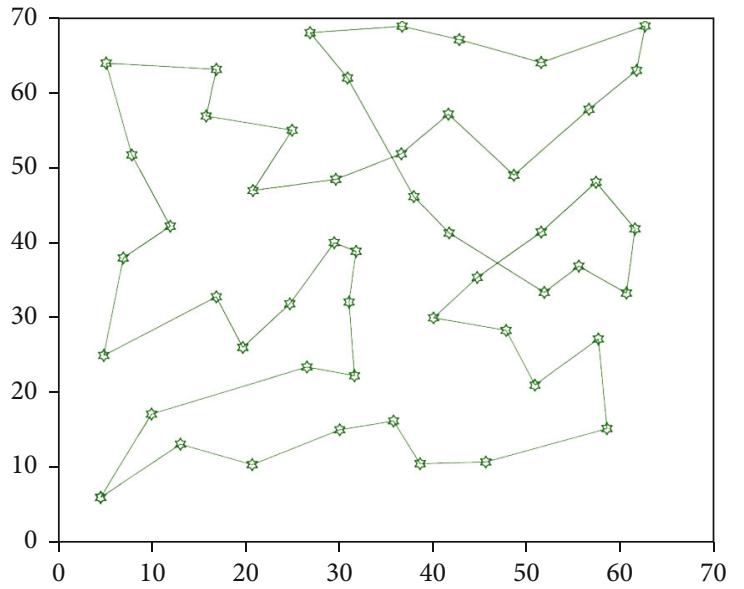

(a) Classic algorithm

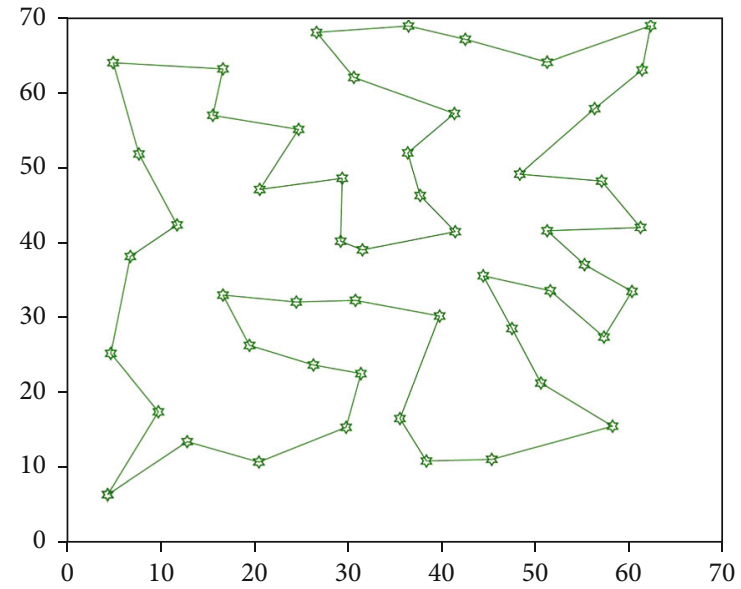

(b) Proposed algorithm

Figure 19: Path planning results solved by two algorithms.

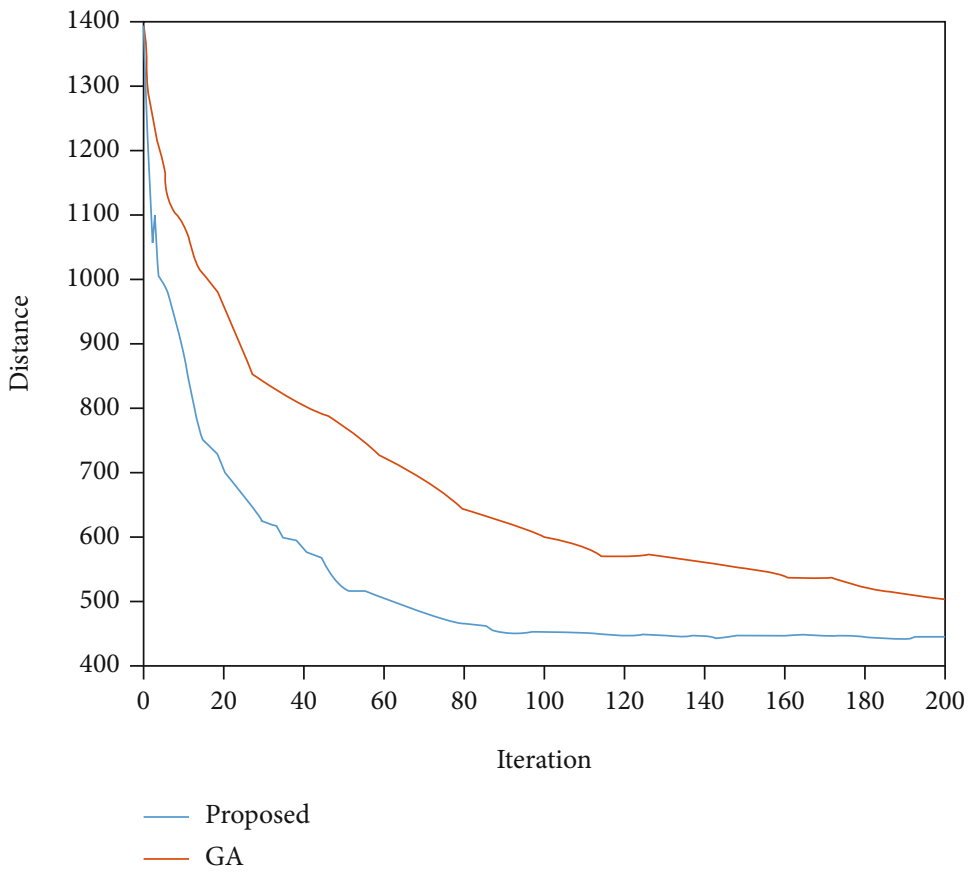

Figure 20: Convergence curve of the proposed algorithm and the classical algorithm.

where $f(x)$ is the objective function of the monitoring path cost described by the chromosome. The smaller objective function value would lead to the larger fitness function value and would be closer to the desired optimal solution.

The new solution space could be completed by crossover operations. First, the father generation was randomly selected, and then position 3 and position 7 were set as the intersection point; the father generation chromosome was cut into three segments and named as the first segment, the middle segment, and the tail segment. To determine the optimal chromosome part of the father generation, these three segments were calculated and compared, and finally, the relatively good offspring was acquired. The flow chart of the entire improved crossover process is shown in Figure 12.

(Step 1) Select p1 and p2 from the father generation randomly, and measure their costs. The cost matrix is shown in Table 1 , and the random generation chromosomes are shown in Figure 13.

(Step 2) Select the first segment of p1, 4-3, and use it as the first segment of offspring 1. Compare the middle segments of $\mathrm{p} 1$ and $\mathrm{p} 2$ to select the 
TABLE 2: Calculation results and contrast for two algorithms.

\begin{tabular}{lccc}
\hline Algorithm & $\begin{array}{c}\text { Convergence } \\
\text { time (s) }\end{array}$ & $\begin{array}{c}\text { Path } \\
\text { cost }\end{array}$ & $\begin{array}{c}\text { Number of } \\
\text { iterations }\end{array}$ \\
\hline Proposed algorithm & 9.2 & 458 & 106 \\
Classic algorithm & 8.8 & 512 & 192 \\
\hline
\end{tabular}

group with the lower cost (for example, in 5-7-6 and 6-1-3, the former that had a lower cost was selected) as the middle section of offspring 1 . Select the tail segment from p2 again to fill in the size of the chromosome. Then, create offspring 2 in the same way. Select the first segment of $\mathrm{p} 2$ to compare, and select the smaller middle segment of $\mathrm{p} 1$ and $\mathrm{p} 2$, and finally select the last segment of $\mathrm{p} 1$. The offspring changes are shown in Figure 14.

(Step 3) Delete the duplicate nodes in the offspring. As shown in Figure 15, delete node 3 of offspring 1 and node 1 of offspring 2 .

(Step 4) Search for the missing node. Insert the missing node 1 in offspring 1 , and compare it with the first and last nodes to acquire the one with a smaller cost. For example, the cost of 1-4 was 46 , and the cost of 2-1 was 29. Therefore, node 1 was inserted after node 2 , and the same steps were applied to offspring 2 . The resulting offspring is shown in Figure 16.

The pseudocode of the algorithm is shown in Algorithm 1, where $N$ is the number of individuals in the population, $G$ is the number of iterations, and $F(t)$ is the fitness value.

\section{Experimental Results and Analysis}

In this chapter, we mainly conducted 4 sets of experiments. First, we conducted computer simulations on the integrated navigation algorithm and path planning algorithm proposed above; second, we controlled the communication distance and conducted experiments on the LoRa communication quality; third, we used unmanned surface vessels for actual surface operations. And we compared and analyzed the actual path and the expected path; fourth, we collected a set of water quality data at the target point in the actual operation and made an analysis of the experimental results.

7.1. Computational Simulations. The computing environment of algorithms is in Win10 Matlab2018b RAM16GB Core (TM) i5CPU.

7.1.1. Simulation of Integrated Navigation Algorithm. Based on the mathematical model of the combined INS/GPS sys-

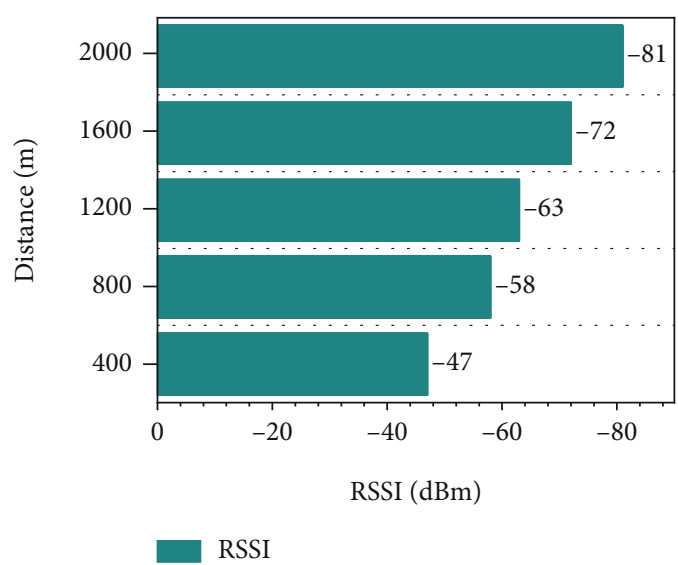

Figure 21: The relationship between transmission distance and RSSI.

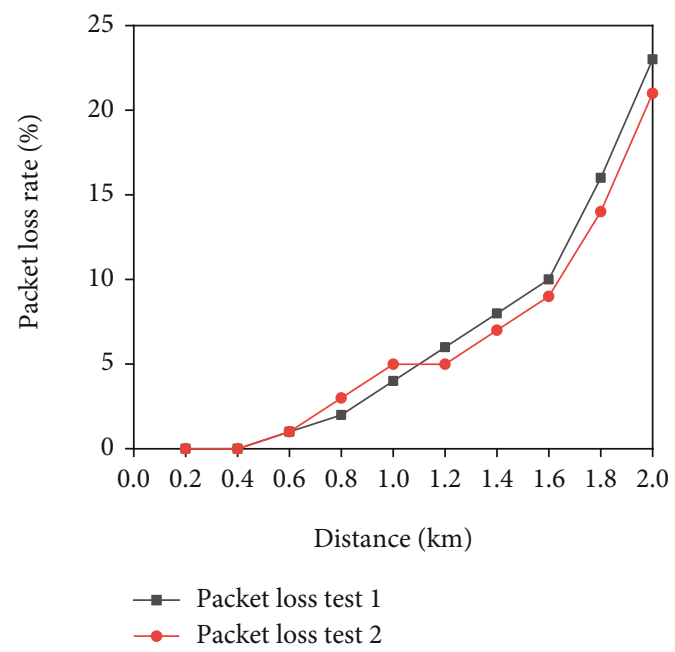

FIgURE 22: The relationship between transmission distance and packet loss probability.

tem designed above, a simulation study was carried out to illustrate the simulation results with actual data. Assume that the first order Markov drift of gyroscope is $30^{\circ} / \mathrm{h}$, the first order Markov zero bias of accelerometer is $0.5 \mathrm{mg}$, the root mean square value of GPS position white noise is $10 \mathrm{~m}$, the root mean square value of GPS velocity white noise is $0.2 \mathrm{~m} / \mathrm{s}$, the root mean square value of random drift of gyroscope is $30 \% \mathrm{~h}$, the root mean square value of random zero bias of accelerometer is $0.5 \mathrm{mg}$, and the simulation time is $500 \mathrm{~s}$. water motion, so the height channel is not simulated in the build simulation. The results are as follows:

The results are shown in Figures 17 and 18, in the presence of noise and zero drift, the GPS correction of the inertial guidance through the Kalman filter is obvious, and the velocity and position can be stabilised within $\pm 1 \mathrm{~m} / \mathrm{s}$ and $\pm 20 \mathrm{~m}$ and remain stable. The feasibility of the optimised combination has been verified and can guide the programming design accordingly. Therefore, the simplified GPS/INS combination can be used by unmanned ships. 
TABLE 3: Table for water quality data of target points.

\begin{tabular}{lccccc}
\hline Node & Coordinate & Temperature $\left({ }^{\circ} \mathrm{C}\right)$ & $\mathrm{pH}$ & Turbidity $(\mathrm{FTU})$ & Conductivity $(\mathrm{s} / \mathrm{m})$ \\
\hline 1 & $118.8861^{\circ} \mathrm{E}, 31.9237^{\circ} \mathrm{N}$ & 7.123 & 7.92 & 187.12 & 82.50 \\
2 & $118.8861^{\circ} \mathrm{E}, 31.9232^{\circ} \mathrm{N}$ & 7.094 & 7.93 & 187.09 & 82.60 \\
3 & $118.8868^{\circ} \mathrm{E}, 31.9234^{\circ} \mathrm{N}$ & 7.013 & 8.04 & 185.33 & 82.30 \\
4 & $118.8869^{\circ} \mathrm{E}, 31.9230^{\circ} \mathrm{N}$ & 7.051 & 7.95 & 185.67 & 82.40 \\
5 & $118.8875^{\circ} \mathrm{E}, 31.9235^{\circ} \mathrm{N}$ & 7.070 & 7.98 & 186.88 & 82.40 \\
6 & $118.8868^{\circ} \mathrm{E}, 31.9242^{\circ} \mathrm{N}$ & 7.106 & 8.02 & 186.72 & 82.50 \\
\hline
\end{tabular}

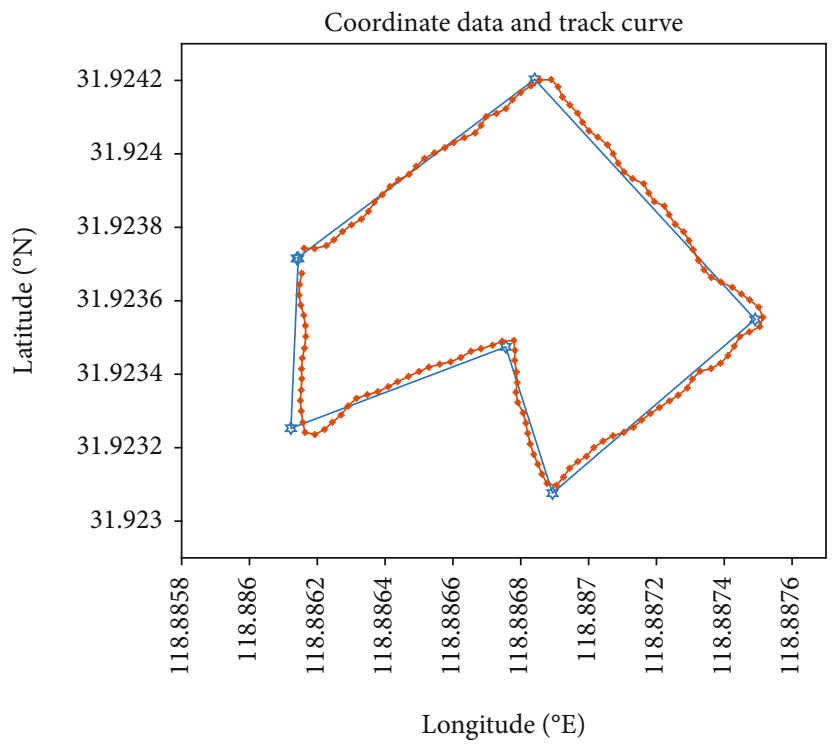

Figure 23: Coordinate data and trajectory curve.

7.1.2. Simulation of Path Planning Algorithm. In order to theoretically verify the effectiveness of the proposed algorithm for the TSP model of water quality monitoring operations, we choose the classical genetic algorithm to compare with the proposed algorithm [16]. The USV passes 50 points in the $70 \times 70$ simulated water area, and then the USV returns to the initial point to form a closed loop. The parameters of the algorithm are set as follows.

(i) Classic algorithm: $\quad M=30, \mathrm{Tm}=200$, Crossover $=$ 0.8 , Mutation $=0.8$

(ii) Proposed algorithm: $M=30, \mathrm{Tm}=200$, Crossover $=$ 0.8 , Mutation $=0.8$

In the above, $M$ is the population size, $\mathrm{Tm}$ is the number of iteration terminations, Crossover is the crossover probability, and Mutation is the mutation probability.

As shown in Figures 19 and 20, the global path planning results and convergence curves corresponding to the two algorithms can be obtained. It is observed that the proposed algorithm quickly converges to the global minimum fitness value in Figure 20. The path calculated by the proposed algorithm is a closed loop, and there is no cross path. Fewer crossing points mean stronger traversal and less travel waste. Figure 20 and Table 2 show that the shortest path distance obtained by the proposed algorithm is 458 , the calculation time of the proposed algorithm is $9.2 \mathrm{~s}$, and the number of iterations is 106. Although the calculation time of the classical genetic algorithm is similar, it does not reach the minimum number of iterations and distance. In short, the proposed algorithm is acceptable in terms of computing time and has better computing performance than the classic algorithm.

7.2. Communication Quality Experiment. The data transmission quality of the USV and the gateway was changed at an interval of $400 \mathrm{~m}$ to test the pack loss probability of the LoRa communication link deteriorates with the distance increasing [17]. Therefore, the RSSI and packet loss probability were tested at different distances in this paper. The data size of each packet was 9 bytes and checked based on CRC, the test distance was $0-2 \mathrm{~km}$, the transmitting power of the radio frequency module was $20 \mathrm{dBm}$, and the antenna gain was $3 \mathrm{dbi}$. 1000 data packets were sent and received for each test distance, and the test was performed twice. The experiment results are shown in Figures 21 and 22.

From Figures 21 and 22, it can be seen that the RSSI of the shipboard LoRa node is greater than $-81 \mathrm{dBm}$ within $2 \mathrm{~km}$, and the signal reception strength is reliable and stable. From the packet loss rate, there is no packet loss within 


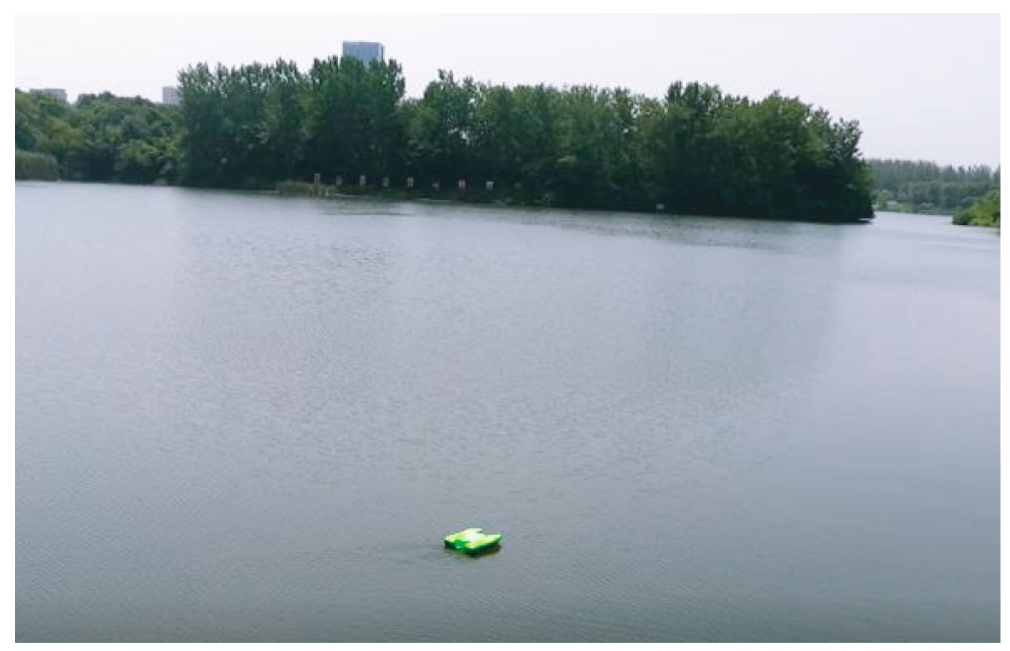

FIgURE 24: Environment of the field test.

$400 \mathrm{~m}$, and the packet loss rate does not exceed 10\% within $1600 \mathrm{~m}$, and the packet loss rate is effectively controlled within 25\% within 1600-2000 m.

Through the above experimental analysis, it can be concluded that the water quality monitoring unmanned boat designed in this paper, based on LoRa network, can achieve a long distance and reliable data transmission link within $2 \mathrm{~km}$, fully enhancing the monitoring range and reliability of the unmanned boat water quality monitoring operation.

7.3. Surface Operation Experiment. In order to verify the reliability of the unmanned surface vessel in actual operation, we set six target monitoring points (these point coordinates are shown in Table 3) in advance in Tianyin Lake of Nanjing Institute of Technology. The user monitoring platform calculates the optimal traversal sequence through the proposed path planning algorithm and sends it to the unmanned surface vessel. After the unmanned surface vessel receives the coordinate set, it traverses one by one and returns to the current coordinate regularly. By fitting the coordinate points, the trajectory diagram of the USV was obtained, as shown in Figure 23.

USV adopts an integrated navigation algorithm based on the Kalman filter and PID heading control algorithm to achieve access to all target detection points. When turning from the current coordinate to the next coordinate, the USV's motion trajectory will show a small deviation, but it can be adjusted to the correct route in time. In short, the unmanned surface vessel designed in this paper can complete the water quality monitoring operation according to the preset path.

7.4. Water Quality Monitoring Results and Analysis. In January 2021, a field test was performed in a local lake. The field test environment is shown in Figure 24.

The USV was set to return its GPS coordinates at an interval of $1 \mathrm{~s}$ and collect water quality information when it arrived at the monitoring point. The water quality data collected at each monitoring point is shown in Table 3.
The experimental site is located in the nature reserve inside the school, and monitoring points 3 and 4 are located in the center of the lake. According to the water quality monitoring results in Table 3 , it can be seen that the turbidity of monitoring points 3 and 4 is lower compared to other monitoring points, and it can be seen that the water in the middle of the lake is clearer, and the water quality of other monitoring points, although slightly worse, is in the normal water quality range, and it can be concluded that the overall water quality of the lake is relatively good.

\section{Conclusion}

To cover the deficiencies of the existing water quality monitoring system, a mobile water quality monitoring system has been designed based on LoRa communication and USV in this paper. The USV in this system is equipped with LoRa nodes and water quality sensors, and the data is transmitted through the LoRa gateway on the shore, by which the realtime monitoring in large areas are realized. Moreover, an improved genetic algorithm is introduced to plan the working path. The test results have proved that the mobile water quality monitoring system designed in this paper can complete the autonomous cruise work in large areas and can monitor the water quality at the target point in real time. Compared with the traditional water quality monitoring mode, the mobile water quality monitoring system proposed in this paper can save costs and labor, enhance working efficiency, and expand the monitoring scope to a great extent.

In the future, we plan to equip the unmanned surface vessel with solar charging panels to improve endurance. And a more complete information-based monitoring platform will be designed around it.

\section{Data Availability}

The data included in this paper are available without any restriction. 


\section{Conflicts of Interest}

The authors declare that there is no conflict of interest regarding the publication of this paper.

\section{Acknowledgments}

This work was supported by the Postgraduate Research \& Practice Innovation Program of Jiangsu Province (No. SJCX20_0698) and School-level Scientific Research Fund of Nanjing Institute of Technology (QKJ201808).

\section{References}

[1] Y. Y. Fu, "Analysis on marine pollution and marine fishery resources protection," Technology Wind, vol. 33, no. 4, p. 133, 2020

[2] T. H. Yang et al., "Development of unmanned surface vehicle for water quality monitoring and measurement," in 2018 IEEE International Conference on Applied System Invention (ICASI), pp. 566-569, Chiba, Japan, April 2018.

[3] R. Yue and T. Ying, "A novel water quality monitoring system based on solar power supply \& wireless sensor network," Procedia Environmental Sciences, vol. 12, no. A, pp. 265-272, 2012.

[4] H. Nam, S. An, C. Kim, S. Park, Y. Kim, and S. Lim, "Remote monitoring system based on ocean sensor networks for offshore aquaculture," in 2014 Oceans - St. John's, pp. 1-7, St. John's, NL, Canada, September 2014

[5] W. Schmidt, D. Raymond, D. Parish et al., "Design and operation of a low-cost and compact autonomous buoy system for use in coastal aquaculture and water quality monitoring," Aquacultural Engineering, vol. 80, pp. 28-36, 2018.

[6] H. Cao, Z. Guo, Y. Gu, and J. Zhou, "Design and implementation of unmanned surface vehicle for water quality monitoring," in 2018 IEEE 3rd Advanced Information Technology, Electronic and Automation Control Conference (IAEAC), pp. 1574-1577, Chongqing, China, October 2018.

[7] S. Siyang and T. Kerdcharoen, "Development of unmanned surface vehicle for smart water quality inspector," in 2016 13th International Conference on Electrical Engineering/Electronics, Computer, Telecommunications and Information Technology (ECTI-CON), pp. 1-5, Chiang Mai, Thailand, June 2016.

[8] M. Bălănescu, G. Suciu, A. Badicu et al., "Study on unmanned surface vehicles used for environmental monitoring in fragile ecosystems," in 2020 IEEE 26th International Symposium for Design and Technology in Electronic Packaging (SIITME), pp. 94-97, Pitesti, Romania, October 2020.

[9] A. Lavric and V. Popa, "Internet of Things and LoRa ${ }^{\mathrm{TM}}$ lowpower wide-area networks: a survey," in 2017 International Symposium on Signals, Circuits and Systems (ISSCS), pp. 1-5, Iasi, Romania, 2017.

[10] Y. Wang, J. Cui, B. Zhao, W. Zhao, and K. Liu, "Observation and communication platform design of USV for marine environmental parameters," in Global Oceans 2020: Singapore U.S, pp. 1-5, Gulf Coast, Biloxi, MS, USA, 2020.

[11] S. L. Yang and D. M. Yang, "Performance advantages of small waterplane area catamaran and its application overview," Marine Technology, vol. 3, pp. 1-4, 2000.
[12] Z. Sun, S. Pan, and L. Yang, "Remote monitoring system for multiple mobile robot based on OneNet cloud platform," in 2018 Chinese automation congress (CAC), pp. 3977-3980, Xi'an, China, 2018.

[13] J. W. Wang, G. M. Dai, B. Q. Xie, and Q. Y. Zhang, "Overview of algorithms for solving TSP problems," Computer Engineering and Science, vol. 36, no. 2, pp. 72-74+155, 2008.

[14] J. Stender, "Introduction to genetic algorithms," in IEE Colloquium on Applications of Genetic Algorithms, pp. 1/1-1/4, London, UK, 1994.

[15] Y. P. Shen, X. J. Zhang, P. Wu, and C. Z. Wang, "Solving traveling salesman problems based on genetic algorithm," Computer CD Software and Applications, vol. 5, no. 10, pp. 21-22+115, 2012.

[16] D. N. Mudaliar and N. K. Modi, "Design and application of $\mathrm{m}$-mutation operator in genetic algorithm to solve traveling salesman problem," in 2019 International Conference on Computation of Power, Energy, Information and Communication (ICCPEIC), pp. 094-096, Melmaruvathur, India, March 2019.

[17] Z. Y. Wang, Z. H. Yu, Q. Q. Wang, and Z. H. Liang, "Study on the coverage and propagation loss of LoRa technology in urban environments," Telecommunications Information, vol. 58, no. 6 , pp. 26-31, 2021. 